\title{
Re-awakening of GRS 1716-249 after 23 yr, observed by Swift/XRT and NuSTAR
}

\author{
Priya Bharali, ${ }^{1,2}$ Sunil Chandra ${ }^{\bullet}, 3,4 \star$ Jaiverdhan Chauhan $^{\oplus}, 4,5 \star$ Javier A. García, ${ }^{6,7}$ \\ Jayashree Roy ${ }^{\odot},{ }^{8,9}$ Markus Boettcher $^{3}$ and Kalyanee Boruah ${ }^{1}$ \\ ${ }^{1}$ Department of Physics, Gauhati University, Guwahati 781014, India \\ ${ }^{2}$ Mahatma Gandhi Government Arts College, Mahe, Puducherry 673311, India \\ ${ }^{3}$ Centre for Space Research, North-West University Potchefstroom, South Africa \\ ${ }^{4}$ Tata Institute of Fundamental Research, Homi Bhabha Road, Mumbai 400005, India \\ ${ }^{5}$ International Centre for Radio Astronomy Research - Curtin University, GPO Box U1987, Perth, WA 6845, Australia \\ ${ }^{6}$ Cahill Center for Astronomy and Astrophysics, California Institute of Technology, Pasadena, CA 91125, USA \\ ${ }^{7}$ Remeis Observatory \& ECAP, Universität Erlangen-Nürnberg, Sternwartstr. 7, 96049 Bamberg, Germany \\ ${ }^{8}$ UM-DAE Center for Excellence in Basic Sciences, University of Mumbai, Kalina, Mumbai 400098, India \\ ${ }^{9}$ Inter-University Center for Astronomy and Astrophysics, Post Bag 4, Pune, Maharashtra 411007, India
}

Accepted 2019 May 28. Received 2019 May 28; in original form 2017 July 18

\begin{abstract}
In this work, we present a spectral and temporal analysis of Swift/XRT and NuSTAR observations of GRS 1716-249 during its recent 2016-2017 outburst. This low-mass X-ray binary underwent an extraordinary outburst after a long quiescence of $23 \mathrm{yr}$, since its last major outburst in 1993. The source was observed over two different epochs during 2017 April 7 and 10. The best-fitting joint spectral fitting in the energy range $0.5-79.0 \mathrm{keV}$ indicates that the spectrum is best described by relatively cold, weak disc blackbody emission, dominant thermal Comptonization emission, and a relativistically broadened fluorescent iron $\mathrm{K} \alpha$ emission line. We observed a clear indication of a Compton hump around $30 \mathrm{keV}$. We also detected an excess feature of $1.3 \mathrm{keV}$. Assuming a lamp-post geometry of the corona, we constrained the inner disc radius for both observations to $11.92_{-11.92}^{+8.62} R_{\mathrm{ISCO}}$ (i.e. an upper limit) and $10.39_{-3.02}^{+9.51} R_{\mathrm{ISCO}}$ (where $R_{\mathrm{ISCO}} \equiv$ radius of the innermost stable circular orbit) for the first epoch (E1) and

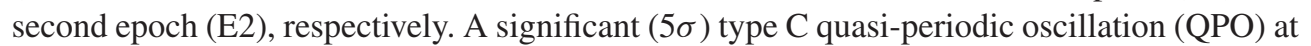
$1.20 \pm 0.04 \mathrm{~Hz}$ is detected for the first time for GRS 1716-249, which drifts to $1.55 \pm 0.04 \mathrm{~Hz}$ $(6 \sigma)$ at the end of the second observation. The derived spectral and temporal properties show a positive correlation between the QPO frequency and the photon index.
\end{abstract}

Key words: accretion, accretion discs - black hole physics - X-rays: binaries - X-rays: individual: GRS 1716-249.

\section{INTRODUCTION}

The rapid time variability of transient black hole X-ray binaries (BHXRBs) provides a unique tool to understand the physical processes like accretion, disc geometry, etc., in the vicinity of the central engine. During X-ray outbursts, BHXRBs undergo various spectral state transitions, namely (i) low/hard (LH), (ii) thermal or high soft (HS), (iii) very high state (VHS)/steep power law (SPL), and (iv) hard and soft intermediate (HIMS and SIMS) states (Belloni et al. 2000; Remillard \& McClintock 2006). The HS state spectrum generally consists of non-thermal flux $<25$ per cent of the total emission, while the thermal emission from the accretion disc

^E-mail: sunil.chandra355@gmail.com (SC); 4u.verdhan@gmail.com (JC) (around the compact object) constitutes the remaining 75 per cent, with either a weak or no quasi-periodic oscillations (QPOs) seen in the light curve. On the other hand, the VHS/SPL exhibits peculiar properties such as the disc flux varying from 35 percent to 80 percent, a photon index $(\Gamma) \geq 2.4$ and a luminosity $(L)$ $>10$ percent of the Eddington luminosity $\left(L_{\mathrm{Ed}}\right)$. Low-frequency QPOs (LF QPOs) are often observed in this state. The LH state is characterized by the disc flux contributing $<20$ percent, and the non-thermal flux dominating at $\geq 80$ percent, a spectral index of $1.4 \leq \Gamma \leq 2.1$, a luminosity $L \leq 1$ per cent of $L_{\mathrm{Ed}}$, and sometimes LF QPOs $(<0.1 \mathrm{~Hz})$ are observed (Remillard \& McClintock 2006).

van der Klis (1989) suggests that the QPO frequencies range from $\mathrm{mHz}$ to $\mathrm{kHz}(\leq 1.2 \mathrm{kHz})$. Based on the peak frequency $(v)$, QPOs are categorized into LF QPOs and high-frequency QPOs (HF QPOs). In the case of BHXRBs, the LF QPOs are characterized by 
$0.1 \leq v \leq 30 \mathrm{~Hz}$. Using the definition of the Quality factor ${ }^{1}$ (also referred to as Q factor), representing the broadness of a QPO peak (Belloni \& Stella 2014), the LF QPOs are further sub-categorized into three types: type A, type B, and type C (Homan et al. 2001; Remillard et al. 2002). Type A LF QPOs are characterized by a weak (few percents of rms amplitude) and broad peak (Q factor $\leq 3$ ) around 7-9 Hz, usually observed on top of weak red noise (Homan et al. 2001; Casella, Belloni \& Stella 2005). Type B LF QPOs represent a relatively strong (4-7 percent rms amplitude) and narrow peak (Q factor $\sim 5-7$ ) between 5 and $7 \mathrm{~Hz}$, usually associated with a weak red noise (few per cent rms) (Motta et al. 2011). The type C LF QPOs are characterized by a strong (up to 21 per cent rms amplitude) and narrow peak ( $Q$ factor $\sim 5-12$ ) with the variable centroid frequency between 0.1 and $15 \mathrm{~Hz}$ superposed on a strong flat-top noise (Wijnands \& van der Klis 1999; Motta et al. 2015).

Different models have been proposed to explain the origin and physical nature of QPOs in X-ray binaries (XRBs). The study of LF QPOs provides an indirect way to understand the accretion flow around the compact object in XRBs. The relativistic precession model (RPM), based on general relativity and proposed by Stella \& Vietri (1998), explains the origin and evolution of LF QPOs and a few HF QPOs in neutron star X-ray binaries. According to this model, QPOs originate as a result of the nodal precession, periastron precession, and Keplerian motion, of a luminous blob of material in the accretion flow around the compact object. Ingram, Done \& Fragile (2009) extended the model proposed by Stella \& Vietri (1998), considering the complete inner flow instead of a luminous blob. The authors tried to demonstrate the origin of LF QPO and the noise linked to them. Later Motta et al. (2014b) extended this model to black hole X-ray binaries.

The Galactic microquasar GRS 1716-249 (or GRO J171924 or Nova Oph 1993) was first detected on 1993 September 25 , independently and simultaneously by the BATSE instrument aboard CGRO (Harmon et al. 1993) and the SIGMA telescope aboard GRANAT (Ballet et al. 1993). The optical counterpart was discovered by della Valle, Mirabel \& Rodriguez (1994) and Masetti et al. (1996), as a low-mass main-sequence star of spectral type K (or later). The mass of the companion is star found to be $1.6 \mathrm{M}_{\odot}$, and the system has an orbital period of $14.7 \mathrm{~h}$. The compact object is believed to be a stellar-mass black hole of mass $4.9 \mathrm{M}_{\odot}$, located at a distance of 2.0-2.8 kpc. The only observed historical major outburst from GRS 1716-249 coincided with its discovery in 1993, which was later studied in detail by van der Hooft et al. (1996), to investigate the nature of its temporal variability. During the entire $80 \mathrm{~d}$ of its outburst, the authors witnessed a QPO at $v \sim 0.04 \mathrm{~Hz}$ at the beginning of the observations, which gradually shifted to $0.3 \mathrm{~Hz}$ at the end. A constant phase lag of $0.072 \pm 0.010$ radian was also observed in the frequency range $0.02-0.20 \mathrm{~Hz}$. van der Hooft et al. (1999) observed that the $\leq 1 \mathrm{~Hz}$ QPO was similar to type II burst profiles of rapid bursters associated with neutron star accretors. This variability feature suggests that the origin of the $\leq 1 \mathrm{~Hz}$ QPO is independent of the nature of the accretor, and the $0.04 \mathrm{~Hz}$ QPO originates from thermal viscous instabilities in the accretion disc surrounding the black hole.

A study of the energy spectra of GRS 1716-249 during the low hard state was presented by Revnivtsev et al. (1996), applying different models for the spectral fitting, such as Comptonization

${ }^{1} \mathrm{Q}$ factor is defined as the ratio between the QPO centroid frequency and the full width at half-maximum of the QPO peak. and optically thin thermal bremsstrahlung. Ling \& Wheaton (2005) carried out a detailed study of spectral variability and the soft $\gamma$-ray flux during a $1000 \mathrm{~d}$ period in 1993-1995.

Using Monitor of All-sky X-ray Image ${ }^{2}$ (MAXI) data, Negoro et al. (2016) reported the first detection of GRS 1716-249 on 2016 December 18 after 23 yr. During the MAXI observation on 2016 December 21, the source was observed with a photon index of $1.62 \pm 0.06$ (Masumitsu et al. 2016). Chandra observations on 2017 February 6 revealed GRS 1716-249 to be in the hard spectral state with a photon index $(\Gamma) \sim 1.53$ (Miller et al. 2017). Based on Swift observations on 2017 March 27 and April 2, Armas Padilla \& Muñoz-Darias (2017) found that the source was transiting to the soft state. However, Swift observations on 2017 May 5 and 11 confirmed that the source had returned to the hard state (Bassi, Del Santo \& Motta 2017). Bassi et al. (2019) studied the 2016-2017 outburst of GRS 1716-249 in the radio and the X-ray bands. Those authors reported that GRS 1716-249 underwent a failed outburst because the source never exhibited the canonical high soft spectral state during this outburst. The radio-X-ray correlation shows that the source is positioned at the radio-quiet 'outlier' branch (Bassi et al. 2019).

This investigation aims to present a spectro-temporal study of GRS 1716-249 during its recent outburst in 2016-2017, using two different observations performed on 2017 April 7 and 10 by the Swift/XRT and NuSTAR observatories. NuSTAR offers a pileup free performance (up to $100 \mathrm{mCrab}$ ), high-energy resolution (400 eV in the range $0.1-10 \mathrm{keV}$ ) and excellent calibration. This instrument provides a rare opportunity to study relativistically skewed iron line profiles and to constrain the inner disc radius with high precision (Harrison et al. 2013; Miller et al. 2013; Pahari et al. 2015; Parker et al. 2015). This paper is organized as follows. Section 2 presents a detailed description of the observations and data reduction methodologies. Section 3 describes our analysis and results of the aforesaid outburst. The last Section 4 presents our discussion and conclusions of the major obtained results.

\section{OBSERVATION AND DATA REDUCTION}

\subsection{NUSTAR FPMA and FPMB}

During the 2016-2017 outburst of GRS 1716-249, NuSTAR observed this source on 2017 April 7 and 10 (Obs. ID:90202055002 and 90202055004). The NuSTAR data were acquired using the two focal plane module telescopes (FPMA and FPMB). The net effective on source exposure times after detector dead-time corrections are 18 and $16 \mathrm{ks}$, respectively. We refer to the two epochs as E1 and E2 in chronological order. The details of the observations can be found in Table 1.

We utilized the standard NUSTAR data analysis software (NUS TARDAS v1.7.1) included in HEASOFT v6.23 along with the calibration database CALDB VERSION 20180419. We used the nupipeline task (version 0.4.6 release date: 2016 October 6) for filtering event files and for making depth corrections from both telescopes. A circular region of radius 100 arcsec centred on GRS 1716-249 was used to extract the source events. For extracting the background events, a circular region of the same size as that of the source with centre 5 arcmin away from the centre of the source was chosen to avoid contamination by the source. Science products, e.g. light curves, energy spectra, response matrix files (rmfs), and

\footnotetext{
${ }^{2}$ http://maxi.riken.jp/top/index.html
} 
Table 1. Details of our Swift/XRT and NuSTAR observations of GRS 1716-249.

\begin{tabular}{|c|c|c|c|c|c|c|c|c|}
\hline $\begin{array}{l}\text { Instrument } \\
\text { name }\end{array}$ & $\begin{array}{l}\text { Observation } \\
\text { ID }\end{array}$ & $\begin{array}{c}\text { Observation } \\
\text { start date } \\
\text { (DD-MM-YYYY) }\end{array}$ & $\begin{array}{l}\text { Observation } \\
\text { start time } \\
\text { (hh:mm:ss) }\end{array}$ & MJD & $\begin{array}{l}\text { Epoch } \\
\text { flag }\end{array}$ & $\begin{array}{l}\text { Effective } \\
\text { exposure } \\
\text { time (ks) }\end{array}$ & $\begin{array}{c}\text { Count rate } \\
\left(\text { cts s }^{-1}\right)\end{array}$ & $\begin{array}{c}\text { Observation } \\
\text { mode }\end{array}$ \\
\hline NuSTAR/FPMA, FPMB & 90202055004 & $10-04-2017$ & $16: 36: 09$ & 57853 & E2 & 16 & $325 \pm 10$ & SCIENCE \\
\hline Swift/XRT & 00034924029 & 07-04-2017 & 08:50:41 & 57850 & E1 & 1.6 & $130 \pm 8$ & WT \\
\hline Swift/XRT & 00034924031 & $10-04-2017$ & $21: 12: 42$ & 57853 & E2 & 1.9 & $145 \pm 8$ & WT \\
\hline
\end{tabular}

auxiliary response files (arfs), for both telescopes (FPMA and FPMB) were generated using the NUPRODUCTS task. Light curves from both telescopes were merged to increase the signal-to-noise $(\mathrm{S} / \mathrm{N})$ ratio. To minimize systematic effects, the energy spectra from both detectors were modelled simultaneously.

\subsection{Swift/XRT}

The Swift/X-ray Telescope (XRT) observations on 2017 April 7 and 10 were utilized for the present study. These observations were either exactly simultaneous (E2; Total Exp. $=1.9 \mathrm{ks}$ ) or nearly simultaneous (E1; Total Exp. = $1.6 \mathrm{ks} ; 5 \mathrm{~h}$ difference from NuSTAR pointing) with the NUSTAR observations discussed in Section 2.1. During both epochs (E1 and E2), XRT operated in windowed timing (WT) mode. For more details about the observations, please refer to Table 1 .

Standard procedures as suggested by the instrument team were followed for the filtering and screening. Swift/XRT data were reduced using the xrtpipeline (version 0.13.2 release date: 2015 January 20). The background-subtracted average count rates during E1 and E2 in WT mode were found to be 130 and 145 counts $\mathrm{s}^{-1}$, respectively. According to Romano et al. (2006), the specified photon pile-up limit of the WT mode data is 100 counts $\mathrm{s}^{-1}$. Therefore, before extracting any scientific product, the possibility of pile-up in the data is tested and corrected following the prescriptions by Romano et al. (2006). Specifically, this was done by investigating the spectral distortion due to pile-up and subsequently removing an appropriate bright portion from the centre of the source image to restore the spectrum. Following this procedure, the most suitable pile-up free source region used for our analysis is represented by a rectangular region defined by $108 \times 36 \operatorname{arcsec}^{2}$, with $21.6 \times 21.6$ $\operatorname{arcsec}^{2}$ removed from the centre. The background region wes chosen to be a similar rectangular region, except 5 arcmin away from the centre along the image strip. The end products, namely energy spectrum and light curves corresponding to the source and background regions were extracted using ftools XSELECT (V2.4d). Afterwards, the XRTMKARF tool was utilized for generating the ARF files using the source spectra and exposure map. The resulting files are then used along with the proper RMF files from the recently updated CALDB, for further spectral analysis.

\section{ANALYSIS AND RESULTS}

\subsection{Temporal analysis}

The publicly available one-day averaged MAXI light curve in the energy range $2.0-20.0 \mathrm{keV}$ is plotted in the left-hand panel of Fig. 1, with hardness ratio as a function of time plotted in the bottom. The hardness ratio is defined as the ratio of count rates in the 4.0-10.0 keV and 2.0-4.0 keV energy bands (Morihana et al.
2013). The 1-d averaged Swift/BAT light curve in the energy band $15.0-50.0 \mathrm{keV}$ is shown in the right-hand panel of the Fig. 1. The long-term light curves from MAXI (2.0-20.0 keV) and BAT (15.0$50.0 \mathrm{keV}$ ), clearly display the overall rising and declining trends in flux. The average count rate over the two epochs E1 and E2 show a small variation as presented in Table 1. For NUSTAR (3.0 $80.0 \mathrm{keV}$ ) the average count rate during E1 is slightly higher than during E2, whereas the reverse trend is seen for the average XRT fluxes.

Fig. 2 shows the 120-s binned light curve corresponding to E1 and E2 for different energy bands. It indicates periodic variations of $5800 \mathrm{~s}$, as derived from folding of the light curve over a range of periods and searching for the maximum chi-square as a function of the period in both observations. However, this could be an artefact of the satellite orbital motion. In order to verify a periodicity of time-scales of 5800 s which nearly matches with the satellite orbital period of NUSTAR, one needs longer observations and more rigorous investigations of the artifacts/systematics introduced by the improper accounting of instrument background etc at different orbital phases of NUSTAR. This anyway is beyond the scope this work and hence will not be discussed.

To characterize the variability in GRS 1716-249 during both epochs E1 and E2, the power density spectrum (popularly known as PDS) was generated. They are displayed in Fig. 3. PDS are generated by using the Fourier transform of the light curves. The peaks in the PDS correspond to the presence of periodic signals in the light curves. The powspec tool distributed as XRONOS sub-package of the heasoft package is used to generate the PDS. The NuSTAR detectors are affected by dead time, which is $2.5 \mathrm{~ms}$ (Harrison et al. 2013; Bachetti et al. 2015). However, we generated PDS for FPMA and FPMB separately, which are not dead-time corrected. The presence of dead time affects the contribution of white noise in the PDS and sometimes affects the overall shape of the QPO, but there is no effect on the QPO peak frequency. A distortion of the overall QPO shape affects the Q factor and significance, but the variation is only significant at higher count rates, above 600 counts $\mathrm{s}^{-1}$ (Bachetti et al. 2015). We observed a QPO in both detectors. The PDSs were fitted with a model consisting of two Lorentzians added together. The first, broader Lorentzian component represents the continuum, whereas the second Lorentzian was used to fit the QPO. The significance of the QPOs was estimated by the ratio of the area under the Lorentzian profile peaking at the QPO frequency (shown with the blue dash-dot-dashed line in Fig. 3) to the $1 \sigma$ negative error in the area estimated by the model. The recipe suggested by Vaughan (2005) was adopted to calculate the confidence level of the detected QPO and to reject low significance peaks.

In Table 2, we present the derived broad Lorentzian knee frequency, QPO frequency, Q factor, its significance and $\chi^{2} /$ dof for various segments of the NUSTAR light curves for both epochs 

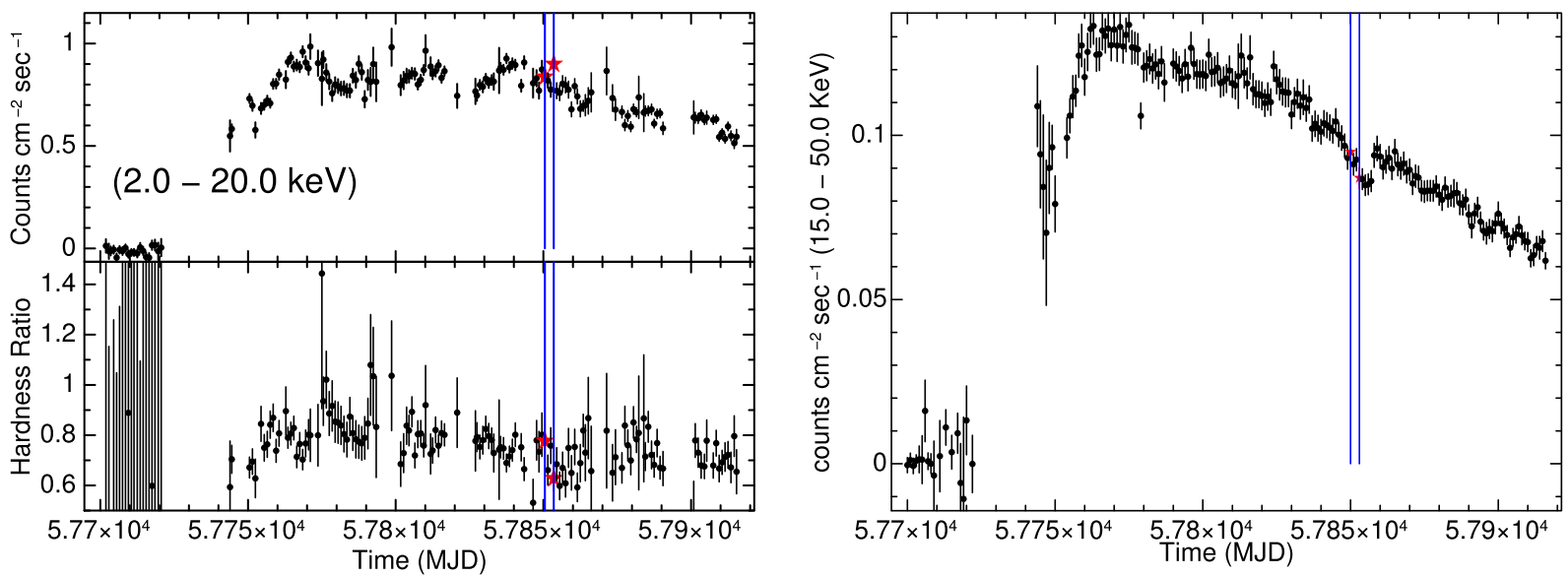

Figure 1. Left: 1-d averaged light curve (top panel) from MAXI (2.0-20.0 keV) along with hardness ratio (bottom panel) to display the long-term variations in GRS 1716-249. Right: The Swift/BAT light curve in the energy range 15.0-50.0 keV. The blue vertical lines indicate the times of the observations used for the present study. Both observations are highlighted with the red star symbol in the MAXI and Swift/BAT plots, and represent the observed flux measured by $M A X I$ and Swift/BAT.
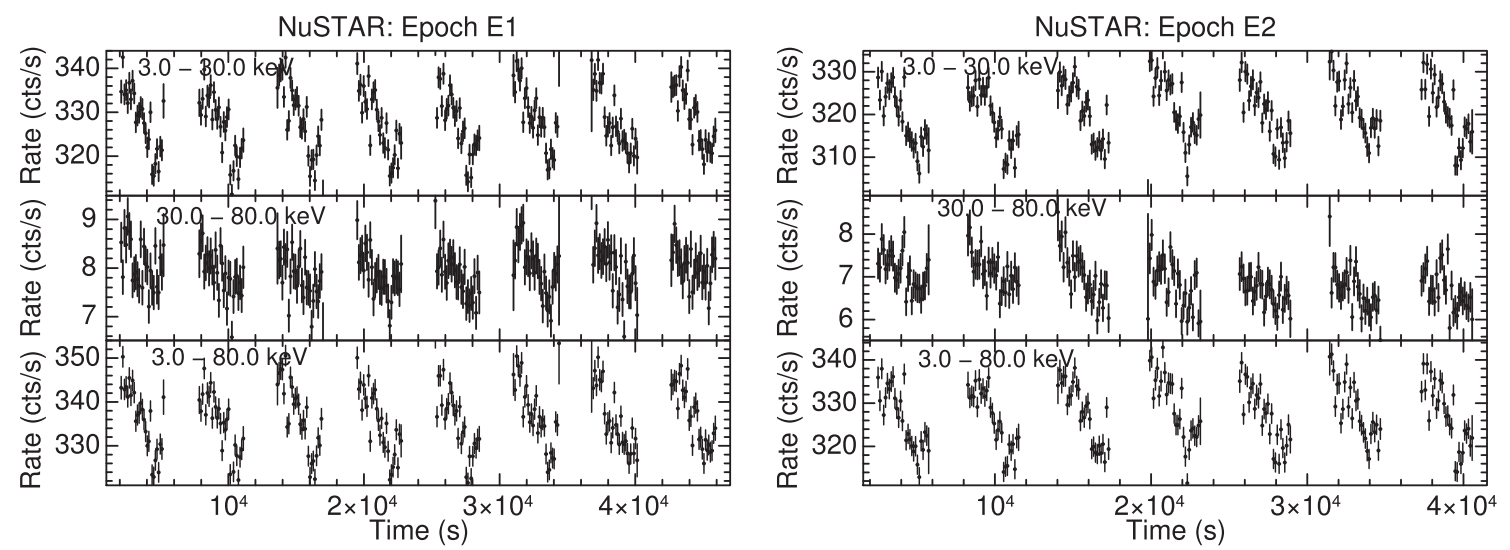

Figure 2. Left: NUSTAR light curves for epoch E1, binned over $120 \mathrm{~s}$, in three different energy bands. The top, middle, and bottom panels correspond to the energy bands 3.0-30.0, 30.0-80.0, and 3.0-80.0 keV, respectively. Right: Same as the left-hand panel, but for epoch E2 (see Table 1 for details of the data). Counts from both FPMA and FPMB are added to improve the S/N ratio.
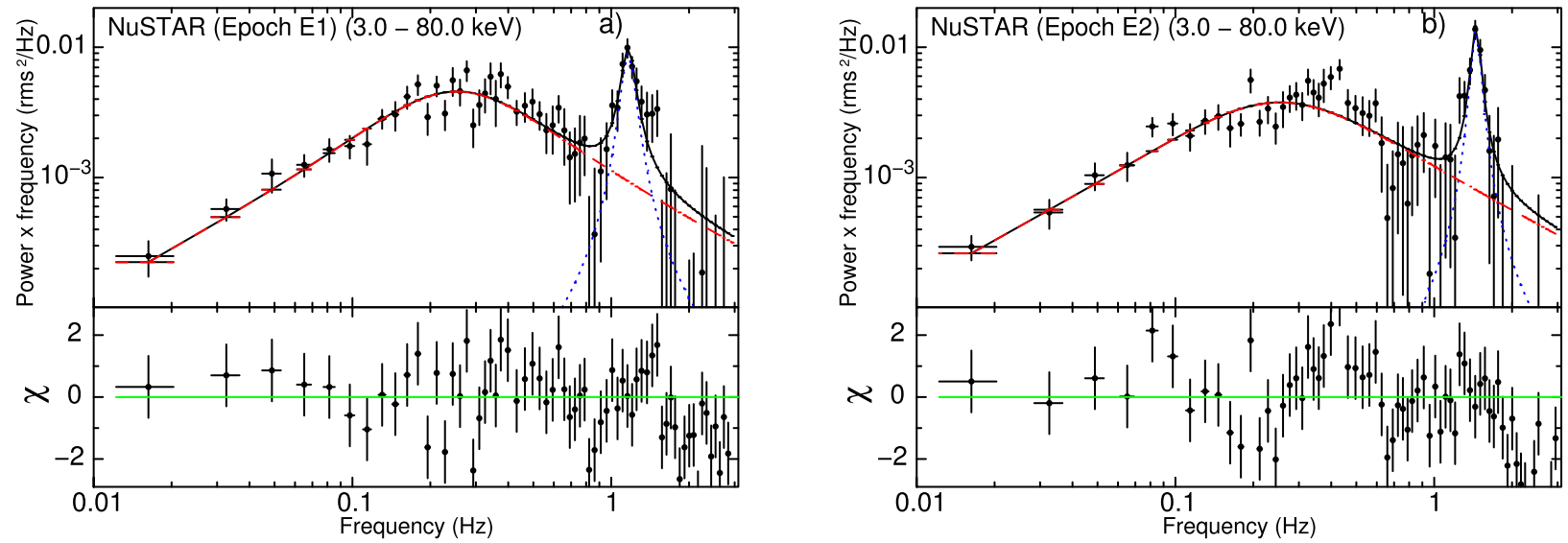

Figure 3. PDS of the light curves in the 3.0-80.0 keV band for epochs E1 (a) and E2 (b), respectively. The bottom panels show the residuals for the same. The red dashed and the blue dotted lines in each plot represent the broad continuum and QPO (peaked at $1.20 \mathrm{~Hz}$ for E1 and $1.55 \mathrm{~Hz}$ for E2) fitted with a Lorentzian model. The solid black line indicates the total model. The observed QPOs have a significance of $6.9 \sigma$ and $8.1 \sigma$, respectively. 
Table 2. Spectral parameters obtained through time-resolved analysis of NUSTAR data.

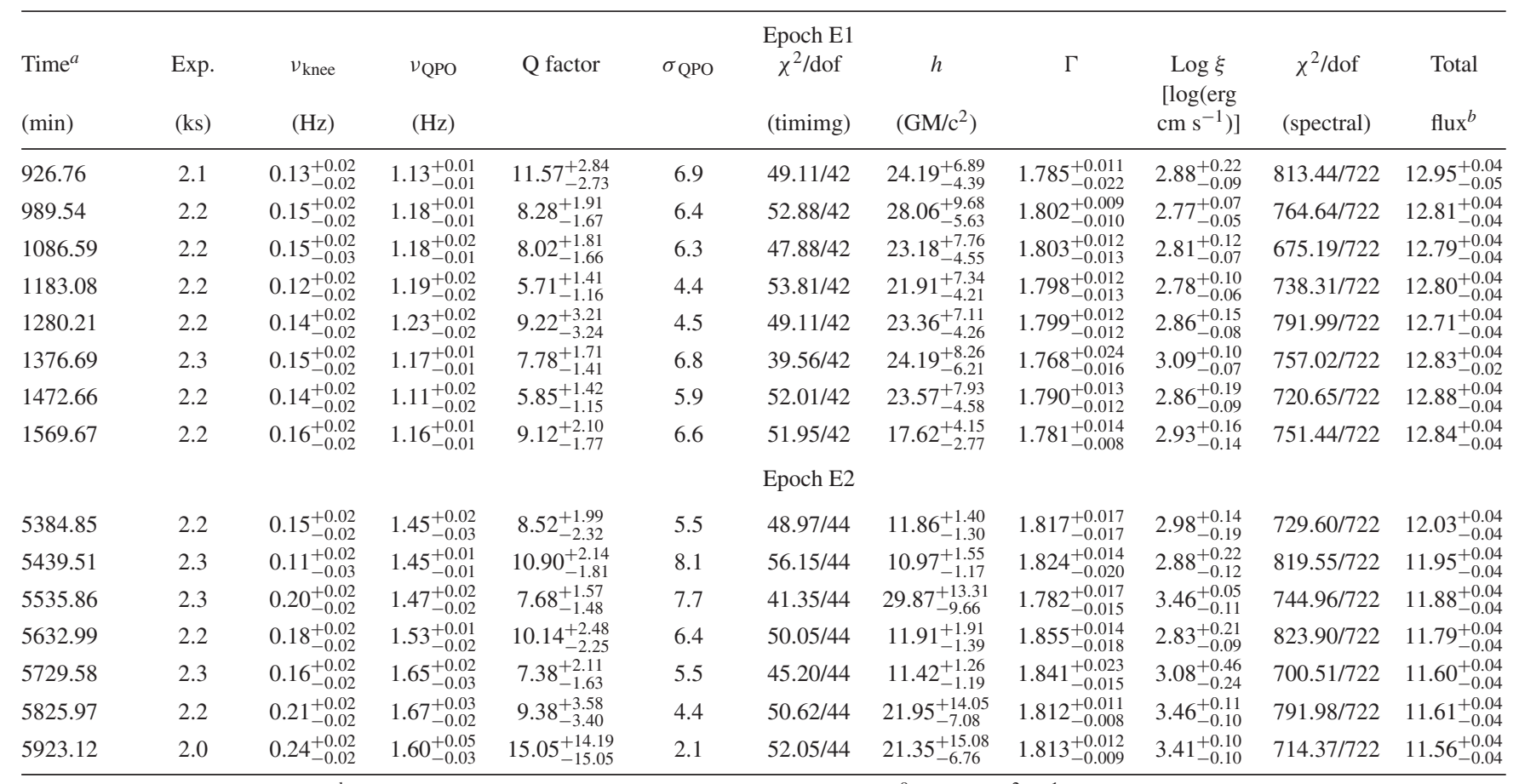

Note. ${ }^{a}$ Time since MJD 57850.0; ${ }^{\mathrm{b}}$ Flux in energy band 3.0-79.0 keV shown in unit of $\left(\times 10^{-9}\right) \mathrm{ergs}^{-2} \mathrm{~s}^{-1}$.

Exp.: exposure time; $v_{\text {noise }}$ : peak frequency of broad Lorentzian noise; $v_{\mathrm{QPO}}$ : peak frequency of QPO; $\sigma_{\mathrm{QPO}}$ : significance of detection; $\Gamma:$ photon Index; log $\xi$ : accretion disc ionization parameter; $h$ : the lamp-post height; $\mathrm{a}^{*}$ is fixed at 0.998 .

in columns 3-7, respectively. The temporal evolution of these parameters is shown in Fig. 4. It is evident from Fig. 4 that there is a hint of variations in the QPO peak frequency $\left(v_{\mathrm{QPO}}\right)$ during both epochs, and we measured a significant change of the QPO peak frequency between the two epochs. During E1, it rises consistently from 1.13 to $1.23 \mathrm{~Hz}$ in $6 \mathrm{~h}$ and afterwards decreases to $1.11 \mathrm{~Hz}$ within the next $3 \mathrm{~h}$, before rising again as evident from the last segment. Although the $\mathrm{Q}$ factor shows some signature of variations, it always remains within $1 \sigma$ uncertainty. The significance of the QPO detection $\left(\sigma_{\mathrm{QPO}}\right)$ was always above 4 . The beginning of E2 witnessed a higher value of $v_{\mathrm{QPO}}$ of $1.45 \mathrm{~Hz}$ which remained constant within $1 \sigma$ for approximately $2.5 \mathrm{~h}$. The next four segments indicate a rising trend for the rest of the observations. $\mathrm{Q}$ factor seems to be constant throughout E2. We also find that the QPO detection is always significant at $\geq 4 \sigma$. Note that the two epochs E1 and E2 are separated by approximately $2.6 \mathrm{~d}$. Therefore, there is an indication that on an average $v_{\mathrm{QPO}}$ is drifting towards higher frequencies with temporary reversals of the trend in between. However, the Q factor shows a small hint of variations with a mean value 8 within $2 \sigma$ errors.

Type C QPOs are strong (rms amplitude 21 per cent), narrow (Q factor $\sim 4-12$ ) with centroid frequency varying within $0.1-15 \mathrm{~Hz}$ and superimposed on a strong flat-top noise (Casella et al. 2005; Pahari, Yadav \& Bhattacharyya 2014). As displayed in Fig. 4 and evident from the PDS fitting parameters listed in Table 2, the QPO frequency varies in the range $1.11-1.67 \mathrm{~Hz}$, the $\mathrm{Q}$ factor ranges within 5-12 and a broad Lorentzian knee frequency is also present in our observations. This confirms that the observed low-frequency QPO to be of type C. The PDS plots displayed in Fig. 3 also confirm the same type of LF QPO. Both the PDS are Leahy normalized and noise subtracted.

\subsection{Spectral analysis}

To understand the spectral characteristics of GRS 1716-249 during the epochs E1 and E2, a simultaneous fit of the broad-band spectrum from NuSTAR/FPMA, FPMB (3.0-78.0 keV) and Swift/XRT (0.5$8.0 \mathrm{keV}$ ) was performed using XSPEC version: 12.10.0 (Arnaud 1996). Before employing more complex models, the spectral fitting was first attempted with individual standard continuum models like diskbb, nthComp, and powerlaw. They generally result in large reduced chi-square values $\left(\chi_{v}^{2}\right)(\gg 2$; i.e. greater than the acceptable limit).

An additional constant multiplicative term was incorporated in the models, utilizing the CONSTANT model inbuilt in XSPEC, to adjust the factors related to the cross-instrument calibration uncertainties. This constant is kept fixed at 1 for Swift/XRT, and the spectra were fitted by keeping it free for FPMA and FPMB. In this way, the best-fitting constants for FPMA and FPMB represent the relative cross-instrument calibration factors with respect to Swift/XRT. This relative cross-instrument factor for E1 was estimated as $1.16 \pm 0.02$ (16 per cent) for both FPMA and FPMB, while for E2 it was found to be $1.08 \pm 0.01$ ( 8 percent) for both telescopes. The recommended acceptable range for the cross-instrument calibration factor between Swift and NuSTAR is 3-20 per cent (Madsen et al. 2015; Marcotulli et al. 2017). Hence, our values clearly fall within the acceptable range as recommended in the literature.

To consistently explain the observed broad-band spectrum, two-component models such as TBabs $\times$ (diskbb + nthComp), TBabs $\times($ diskbb + powerlaw), and TBabs $\times$ (nthComp + powerlaw) were further fitted to the data. The observed spectrum at both epochs (E1 and E2) were found to be reasonably well described by the 

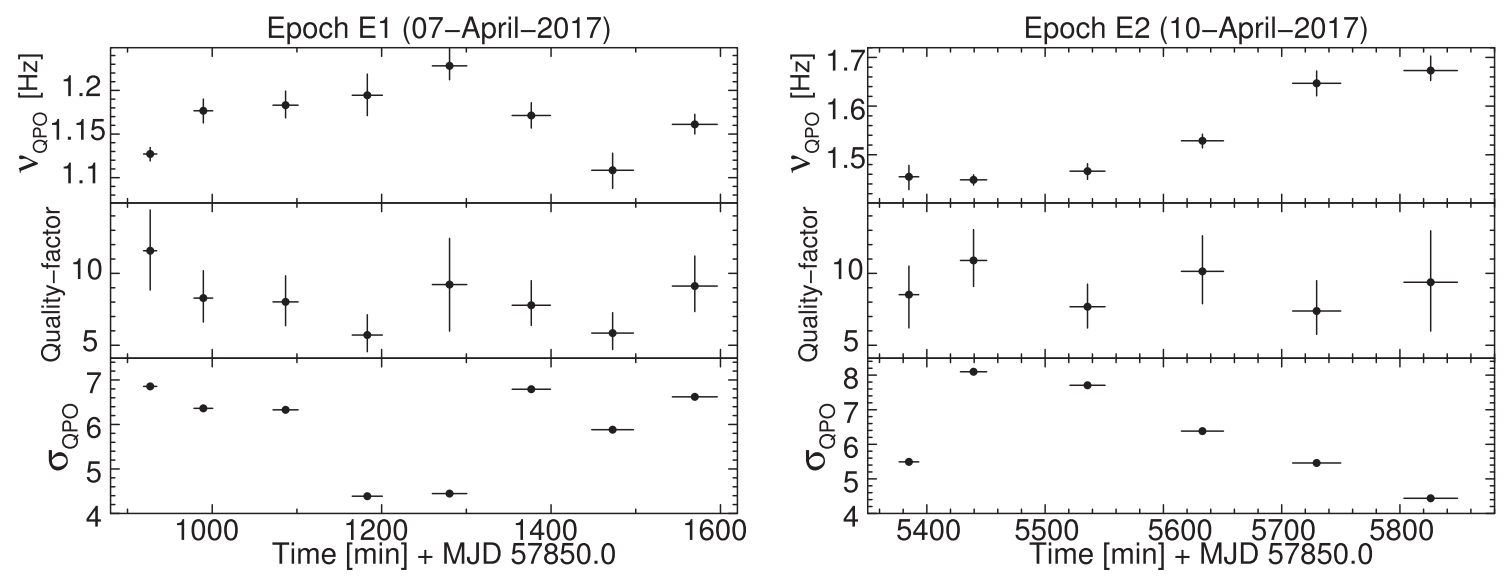

Figure 4. Left: Temporal evolution of the QPO frequency (top panel), Quality factor (middle panel) and QPO significance ( $\sigma$; bottom panel) derived from the NUSTAR observation dated 07 April 2017. Right: Same as in the left-hand panel, but for the NuSTAR observation on 2017 April 10. The QPO frequency clearly shows significant variations in the two epochs, whereas the $\mathrm{Q}$ factor remains constant within uncertainties. The significance of the $\mathrm{Q}$ factor varies, but is always above $4 \sigma(4 \sigma-7 \sigma$ for $\mathrm{E} 1$ and $4 \sigma-8 \sigma$ for $\mathrm{E} 2)$.

TBabs $\times($ diskbb + nthComp $)$ model, i.e. the combination of multicoloured disc blackbody component (diskbb: Mitsuda et al. 1984; Makishima et al. 1986) and a thermal Comptonization model (nthComp : Zdziarski, Johnson \& Magdziarz 1996; Zỳcki, Done \& Smith 2009) modulated by Galactic absorption by neutral hydrogen (TBabs: Wilms, Allen \& McCray 2000). We found excesses around 1.3, 6.4, and $30 \mathrm{keV}$ as shown in Fig. 5. An excess around $6.4 \mathrm{keV}$ is indicative of an $\mathrm{Fe}$ emission line from the accretion disc, while the observed residuals around $30 \mathrm{keV}$ indicate a Compton hump, which, together with the relativistically broadened $\mathrm{Fe} \mathrm{K} \alpha$ line, is typical for Compton reflection. A soft excess around $1.3 \mathrm{keV}$ is typically observed when the surface of the accretion disc is ionized. In this case, electron scattering becomes important along with the re-emission of emission lines, causing a soft excess (Ross \& Fabian 1993, 2005; García et al. 2013). We found that the red wing of the $\mathrm{Fe}$ emission line is extended down to $6 \mathrm{keV}$ while the blue wing is stretched close to $8 \mathrm{keV}$ with a dip around $11 \mathrm{keV}$ as shown in Fig. 5.

Therefore, we replaced the nthComp model by the selfconsistent, broad-band reflection model relxilllpcp from the relxill (v1.0.2: Dauser et al. 2014; García et al. 2014) model family, to account for the relativistic reflection spectrum. Thus, our best-fitting model is TBabs $\times($ diskbb + relxilllpCp). The relxilllpCp model is based on a lamp-post geometry of the corona, which is believed to be the illuminating source. In the lamppost geometry, the corona is treated as a point source positioned at a height $h$, on the black hole spin axis above the accretion disc. The relxilllpcp model uses the thermal Comptonization model nthComp as the input continuum. In the case of the relxilllpCp model, the value of the reflection fraction ( $\left.\operatorname{Refl}_{\text {frac }}\right)$ can be self-consistently determined, depending on the values of the lamp-post height $(h)$, the black hole spin parameter $\left(\mathrm{a}^{*}\right)$ and the inner accretion disc radius $\left(R_{\text {in }}\right)$ through ray-tracing calculations. The evaluation of $\mathrm{Refl}_{\text {frac }}$ by the model itself helps to reduce the parameter space and eventually constrains the geometry of the system (Dauser et al. 2014).

While fitting the average spectra for both epochs using best-fitting model (i.e. TBabs $\times$ (diskbb + relxilllpcp)), we fixed the value for the outer radius of the accretion disc at $400 r_{\mathrm{g}}$, where $r_{\mathrm{g}}$ is the gravitational radius $\left(r_{\mathrm{g}} \equiv \mathrm{GM} / \mathrm{c}^{2}\right)$. We simultaneously fitted for a* and $R_{\text {in }}$ but those two parameters are degenerate, and the effective inner accretion disc radius is controlled by both $\mathrm{a}^{*}$ and the disc inclination angle. In the case of a non-rotating black hole $\left(\mathrm{a}^{*} \approx 0\right), R_{\mathrm{ISCO}} \equiv 6 r_{\mathrm{g}}$. However, in the case of a Kerr black hole, considering the co-rotating case where the accretion disc is rotating in a direction same as the compact object, $R_{\mathrm{ISCO}} \equiv r_{\mathrm{g}}$ (Bardeen, Press \& Teukolsky 1972; Thorne 1974). During the spectral fitting for both E1 and E2, the spin parameter $\left(\mathrm{a}^{*}\right)$ approached the hard upper limit of 0.998 . Therefore, we have frozen the value of $\mathrm{a}^{*}$ at 0.998 and kept $R_{\text {in }}$ free to vary. For a black hole of $\mathrm{a}^{*} \approx 0.998, R_{\mathrm{ISCO}}$ $\approx 1.24 r_{\mathrm{g}}$ (Bardeen et al. 1972; Thorne 1974).

Additionally, to determine the real nature of the system, i.e. whether the compact object is favouring a low spin or a truncated disc, we fixed the value of $R_{\text {in }}$ at the ISCO and allowed the spin parameter to vary. We found that $\mathrm{a}^{*}$ continued to saturate at the maximum value and the $2 \sigma$ uncertainty on the spin parameter provided a lower bound of 0.73 . It is observed that the $\chi^{2} /$ dof is 949.04/906 if we freeze $\mathrm{a}^{*}$ at its maximum value (0.998), whereas it is $1010.72 / 906$ when we fix $R_{\text {in }}$ at the ISCO. This implies that the system prefers a truncated disc over a low spin.

During our analysis for both epochs, we were unable to constrain the electron temperature $\left(\mathrm{kT}_{\mathrm{e}}\right)$. Therefore, we fixed it at a value of $400 \mathrm{keV}$. From our spectral fittings, the iron abundance $\left(A_{\mathrm{Fe}}\right)$ was found to be $0.79_{-0.05}^{+0.11}$ times the solar abundance for E1. However, for E2 the value of the iron abundance is found to be $1.01_{-0.08}^{+0.34}$ times the solar abundance. Apart from the above-mentioned parameters, we determined the following reflection and continuum parameters using the relxilllpcp model: the lamp-post height $(h)$, the power-law index $(\Gamma)$, the $R_{\text {in }}$, the ionization index of the accretion $\operatorname{disc}(\log \xi)$ and the flux due to reflection.

Even after using the best-fitting model $($ TBabs $\times($ diskbb + relxilllpCp) $)$, we have observed a small excess around 6-7 keV in the XRT spectra. The main reason for this excess could be cross-calibration errors. The position of the line is better constrained by NUSTAR spectrum in comparison to the Swift/XRT spectrum, due to the high S/N ratio for NuSTAR, owing to its excellent sensitivity in this energy range. The iron line profiles observed by Swift/XRT and NUSTAR do not match perfectly for the same reason, and hence identical constraints for the Gaussian profile of the line for both instruments cannot be obtained by simultaneous fitting. Therefore, a small excess around $6-7 \mathrm{keV}$ is visible in the Swift/XRT spectrum. The equivalent widths of the 

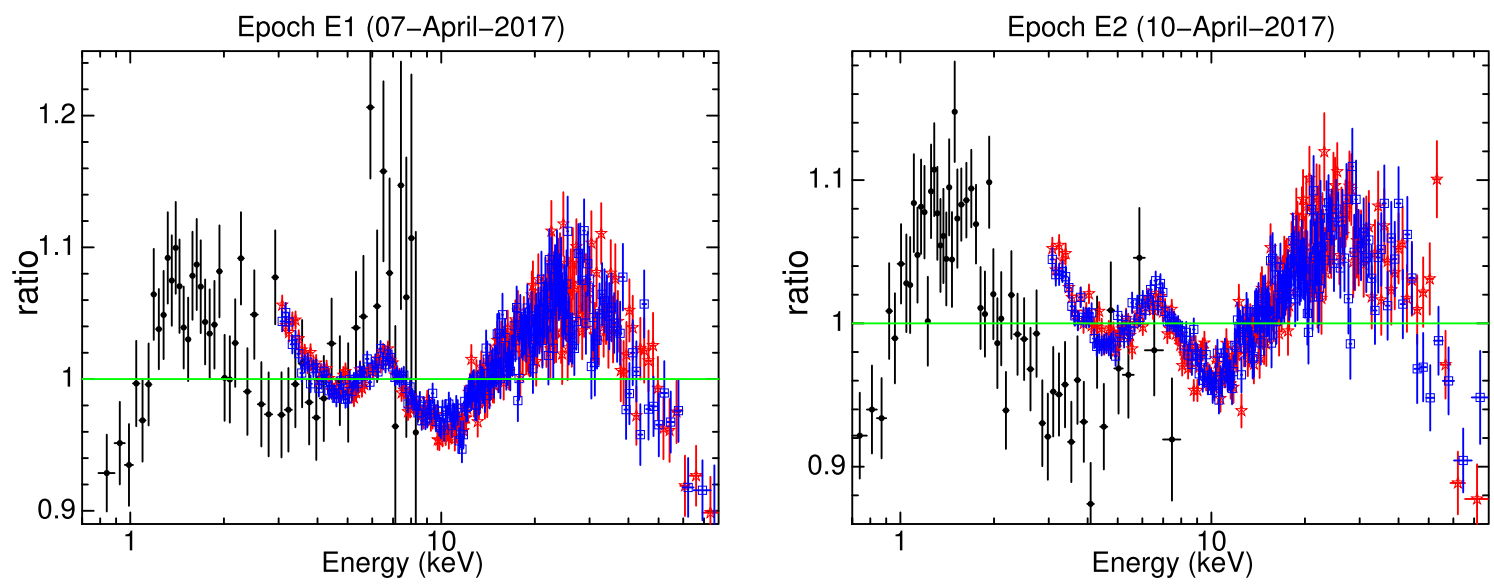

Figure 5. Left: Ratio of jointly fitted Swift/XRT (black dots) and NuSTAR FPMA (red star)/FPMB (blue square) spectra for epoch E1. The spectra are fitted using a simple phenomenological model TBabs $\times$ (discbb + nthComp) for observation $\mathrm{E} 1$ in the energy range $0.5-79 \mathrm{keV}$. A broad emission line feature in the energy range 5-8 keV and a dip around $11 \mathrm{keV}$ is significantly detected. Around 1 and $30 \mathrm{keV}$ a hump-like excess is also observed. Right: Same as on the left, but for epoch E2.

$6.4 \mathrm{keV}$ line derived from simultaneous fitting, corresponding to the epochs (E1) and (E2), are found to be $0.222 \pm 0.001 \mathrm{keV}$ and $0.173 \pm 0.001 \mathrm{keV}$, respectively.

The fitted spectra along with the different model components and residuals, for both epochs (E1 and E2) are presented in the left- and right-hand panel of Fig. 6 (see Table 3 for the best-fitting parameters), respectively. For the broad-band continuum, we used the TBabs (Wilms et al. 2000) model inbuilt in XSPEC to account for the Galactic neutral hydrogen column density. The most recent abundance model (aspl: Asplund et al. 2009), inbuilt in XSPEC, was adopted as an abundance input for the TBabs model. The best-fitting absorption column density, $n_{\mathrm{H}}$ (in units of $10^{22} \mathrm{~cm}^{-2}$ ) was found to be $0.62_{-0.02}^{+0.02}$ and $0.59_{-0.01}^{+0.01}$ for the epochs E1 and E2, respectively. These values show significant excess over the Galactic value in the direction of source as estimated by the online tools of the LAB Survey ${ }^{3}$ (Kalberla et al. 2005) $\left(n_{\mathrm{H}}=0.26 \times 10^{22} \mathrm{~cm}^{-2}\right)$ implying the presence of intrinsic excess absorption in this BHXRB system.

The disc temperature ( $T_{\text {in }}$ of the diskbb model) shows a small variation but the ionization index of the accretion disc $(\log \xi)$, as well as the lamp-post height $(h)$ as estimated from the broad-band spectral fitting at E1 and E2, do not show any significant change. The power-law index $(\Gamma)$ increases from $1.768_{-0.006}^{+0.013}(\mathrm{E} 1)$ to $1.812_{-0.006}^{+0.006}$ (E2). This increment in $\Gamma$ suggests that the source is drifting towards the soft state. The obtained inner disc radii $R_{\text {in }}\left(r_{\mathrm{g}}\right)$ for E1 and E2 are $14.78_{-14.78}^{+5.93} r_{\mathrm{g}}$ and $12.88_{-3.58}^{+5.06} r_{\mathrm{g}}$, respectively. The disc ionization $(\log \xi)$ is high for both observations and its values corresponding to E1 and E2 are found to be $3.07_{-0.03}^{+0.06}$ and $3.19_{-0.07}^{+0.28}$, respectively. The best-fitting lamp-post height $(h)$ is $23.77_{-10.84}^{+16.02}$ and $12.75_{-5.11}^{+7.30}$ in units of $r_{\mathrm{g}}$. The variation in $\mathrm{h}$ is small and within $2 \sigma$ error bars. The $2 \sigma$ error in all the above-mentioned quantities were calculated using the error command in XSPEC.

In order to constrain the inner radius of the accretion disc from our best-fit model (TBabs $($ diskbb + relxilllpCp)), we determined $\Delta \chi^{2}$ using steppar command in XSPEC. The variation of the resulting $\Delta \chi^{2}$, while changing the inner disc radius as the free parameter between $1 R_{\mathrm{ISCO}}$ and $23 R_{\mathrm{ISCO}}$ for epochs E1 and E2, is illustrated in the left-hand panel of Fig. 7. The $2 \sigma$ and

${ }^{3}$ https://www.astro.uni-bonn.de/hisurvey/profile/index.php
$3 \sigma$ significance levels are shown by the horizontal lines. Within $3 \sigma$ bounds, the value of the inner disc radius for the epochs E1 and E2 are found to be $11.92_{-11.92}^{+8.62} R_{\mathrm{ISCO}}$ and $10.39_{-3.02}^{+9.51} R_{\mathrm{ISCO}}$ or $14.78_{-14.78}^{+10.69} r_{\mathrm{g}}$ and $12.88_{-3.74}^{+8.50} r_{\mathrm{g}}$, respectively. The first epoch (E1) represents only an upper bound. Similarly, we have also constrained the disc inclination angle as shown in the right-hand panel of Fig. 7. The value of the inclination angle is found to be $54.19_{-12.48}^{+7.43}$ and $46.59_{-10.38}^{+11.78}$ for $\mathrm{E} 1$ and $\mathrm{E} 2$, respectively, within $3 \sigma$ bounds.

The un-absorbed total fluxes corresponding to the different continuum (disc and Comptonized emission) components have significantly changed from E1 to E2. For example, the total flux has increased by 5 percent after considering the proper error propagation. As mentioned earlier, the source was in a relatively softer state at E2 in comparison to E1. The detailed parameters obtained from modelling the broad-band X-ray spectrum (for both epochs E1 and E2) are summarized in Table 3. We have witnessed significant changes in $\Gamma$, making the overall spectra during E2 relatively softer than during $\mathrm{E} 1$. This implies the possibility of successive changes in $\Gamma$, i.e. its evolution during the spectral state change of GRS 1716-249 from E1 to E2.

Aiming to study this behaviour, the overall NuSTAR light curves during E1 and E2 were subdivided into 8 and 7 fragments, respectively. The same segments were used for investigating the QPOs and the spectral fittings. Due to the unavailability of data at low energies $(<3.0 \mathrm{keV})$ during these fragments, we have modelled only the $3.0-79.0 \mathrm{keV}$ band. Absorption by neutral hydrogen is modifying the continuum at low energies and in our observations the disc is weak, extending only up to $3.5 \mathrm{keV}$. Hence, the NuSTAR data cannot constrain the disc contribution. Therefore, we have kept $n_{\mathrm{H}}$ and $T_{\text {in }}$ fixed to the values obtained after fitting the overall average spectrum (listed in Table 3) for both epochs E1 and E2. However, we can assume that certain parameters do not change significantly over a time-scale as small as $2 \mathrm{ks}$. Therefore, we have fixed $R_{\text {in }}$ and $A_{\mathrm{Fe}}$ to their respective values obtained after fitting the overall average spectrum of epoch E1 and E2, as detailed in Table 3 .

The results from the above-mentioned time-resolved spectral fitting are shown in Fig. 8 and are tabulated in Table 2. Note that the error bars shown in Fig. 8 represent $2 \sigma$ errors. This clearly shows that the spectral parameters, namely $\Gamma$ and $\log \xi$ derived for both E1 and E2 show a hint of variation within $2 \sigma$ significance. $\Gamma$ shows an overall softening from epoch E1 to E2. On the other hand, the lamp- 

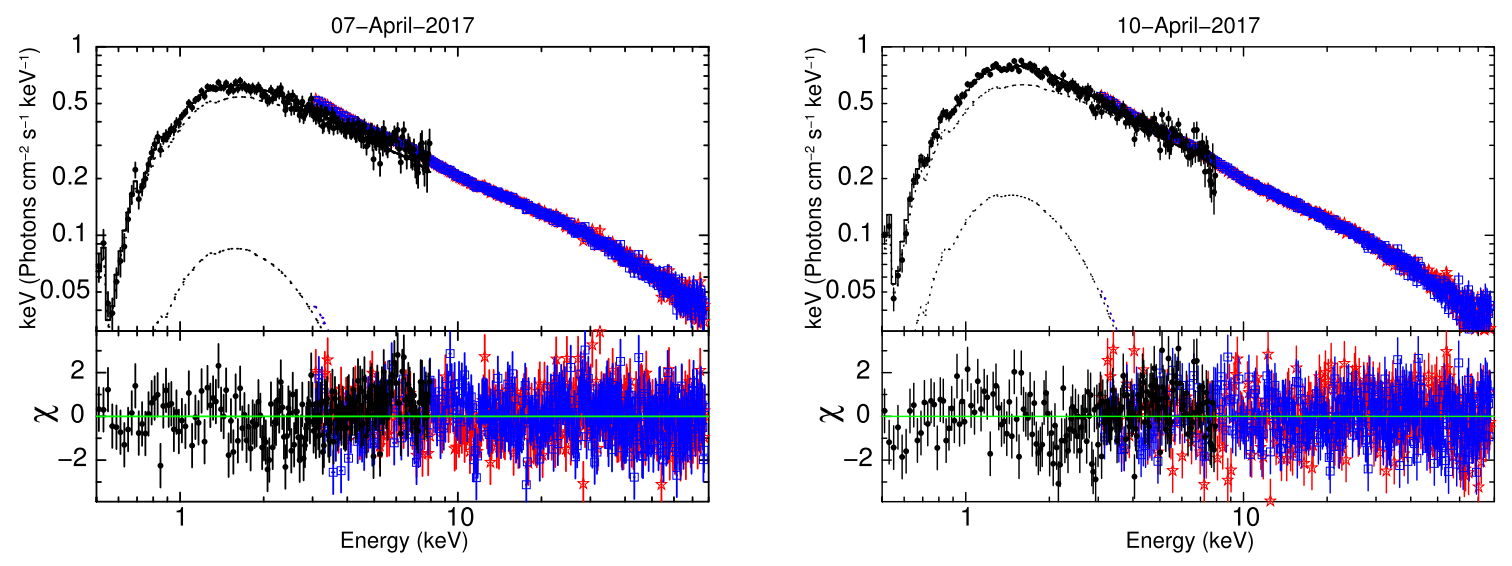

Figure 6. Swift/XRT (black dots) and NuSTAR FPMA (red star)/FPMB (blue square) unfolded spectra of GRS 1716-249 along with the best-fitting model components (TBabs $\times($ diskbb $+\operatorname{relxill}(l p) \mathrm{Cp})$ ), and residuals for epoch E1 (left-hand panel) and epoch E2 (right-hand panel). a* is fixed at 0.998 .

Table 3. Model parameters from simultaneous Swift/XRT (0.5-8.0 keV) and NuSTAR (3.0-79.0 keV) spectral fitting. The model which provides the best fit is TBabs $\times$ (diskbb + relxill (lp) Cp). $2 \sigma$ errors are quoted. This model resulted in $\chi^{2} / \mathrm{dof}=949.04 / 906$ and 1097.55/906 for E1 and E2, respectively. Fig. 6 shows the fitted energy spectrum along with the model constituents and residuals for both observations.

\begin{tabular}{|c|c|c|c|}
\hline Component & Parameter & $\begin{array}{c}\text { Epoch } \\
\text { E1 }\end{array}$ & $\begin{array}{c}\text { Epoch } \\
\text { E2 }\end{array}$ \\
\hline TBABS & $N_{\mathrm{H}}\left(\times 10^{22} \mathrm{~cm}^{-2}\right)$ & $0.62_{-0.02}^{+0.02}$ & $0.59_{-0.01}^{+0.01}$ \\
\hline diskbb & $\begin{array}{c}T_{\text {in }}(\mathrm{keV}) \\
\text { Norm. }\end{array}$ & $\begin{array}{c}0.70_{-0.04}^{+0.06} \\
132.18_{-49.11}^{+53.73}\end{array}$ & $\begin{array}{c}0.59_{-0.02}^{+0.02} \\
437.70_{-89.48}^{+105.49}\end{array}$ \\
\hline \multirow[t]{11}{*}{ relxill(lp)Cp } & $h\left(\mathrm{GM} / \mathrm{c}^{2}\right)$ & $23.77_{-10.84}^{+16.02}$ & $12.75_{-5.11}^{+7.30}$ \\
\hline & $\begin{array}{c}\mathrm{a}^{*}\left(\mathrm{cJ} / \mathrm{GM}^{2}\right) \\
i(\mathrm{deg})\end{array}$ & $\begin{array}{c}0.998^{f} \\
54.19_{-7.89}^{+6.77}\end{array}$ & $\begin{array}{c}0.998^{a} \\
46.59_{-585}^{+3.83}\end{array}$ \\
\hline & $\mathrm{R}_{i n}^{b}\left(R_{\mathrm{ISCO}}\right)$ & $11.92_{-11.92}^{+4.78}$ & $10.39_{-2.89}^{+4.08}$ \\
\hline & $\Gamma$ & $1.768_{-0.006}^{+0.013}$ & $1.812_{-0.006}^{+0.006}$ \\
\hline & $\log \xi\left[\log \left(\mathrm{erg} \mathrm{cm} \mathrm{s}^{-1}\right)\right]$ & $3.07_{-0.03}^{+0.06}$ & $3.19_{-0.07}^{+0.28}$ \\
\hline & $A_{\mathrm{Fe}}($ solar $)$ & $0.79_{-0.05}^{+0.11}$ & $1.01_{-0.08}^{+0.34}$ \\
\hline & Norm. $\left(\times 10^{-2}\right)$ & $3.11_{-0.32}^{+0.56}$ & $3.17_{-0.34}^{+0.85}$ \\
\hline & $F_{\text {Total }}\left(\times 10^{-9} \mathrm{ergs} \mathrm{cm}^{-2} \mathrm{~s}^{-1}\right)$ & $14.85_{-0.16}^{+0.16}$ & $15.59_{-0.14}^{+0.14}$ \\
\hline & $F_{\text {diskbb }}\left(\times 10^{-9} \mathrm{ergs} \mathrm{cm}^{-2} \mathrm{~s}^{-1}\right)$ & $0.49_{-0.05}^{+0.05}$ & $0.89_{-0.07}^{+0.08}$ \\
\hline & $F_{\text {relxill }}\left(\times 10^{-9} \mathrm{ergs} \mathrm{cm}^{-2} \mathrm{~s}^{-1}\right)$ & $14.40_{-0.15}^{+0.15}$ & $14.88_{-0.14}^{+0.14}$ \\
\hline & $\chi^{2} / \mathrm{dof}$ & $949.04 / 906$ & $1097.55 / 906$ \\
\hline
\end{tabular}

Notes: ${ }^{a}$ Inner disc radius; ${ }^{b}$ tags imply that the specific parameter was frozen to these values while fitting the spectra.

(1) Note that all the errors are calculated using error command in XSPEC.

(2) The flux values shown in this table are unabsorbed and calculated for the energy band $0.5-79.0 \mathrm{keV}$.

post height (h) is almost invariable during both epochs (E1 and E2) within $2 \sigma$ uncertainties. When we compare the spectral parameters obtained from time-resolved spectrum fitting and average spectrum fitting, we see that there is some variation in the values of $\Gamma$ and $\log \xi$. Some $\Gamma$ values are above the average values, whereas the $\log$ $\xi$ values are below the average value. This variation in $\Gamma$ and $\log$ $\xi$ could be due to the difference in the energy range between the average spectrum and the time-resolved spectrum. For the average spectrum, we performed a fit in the energy range $0.5-79.0 \mathrm{keV}$, whereas we carried out time-resolved spectrum fitting in the energy range 3.0-79.0 keV, as we do not have Swift/XRT data for each time interval. A systematically lower $\log \xi$ corresponds to a larger $\Gamma$ to produce a similar fit. These two parameters are anticorrelated to some degree and hence, if $\Gamma$ systematically increases, the ionization parameter will decrease (García et al. 2013; Choudhury et al. 2017).

\subsection{Correlation study}

The correlations between the model parameters derived from the time-resolved spectroscopy and the QPO frequency $\left(v_{\mathrm{QPO}}\right)$ were also studied. The Pearson correlation test is performed to quantify the correlation, using the following definition:

(i) Pearson correlation coefficient (Pearson 1920)

$r=\frac{\Sigma(x-\bar{x})(y-\bar{y})}{\sqrt{\Sigma(x-\bar{x})^{2}} \sqrt{\Sigma(y-\bar{y})^{2}}}$, 

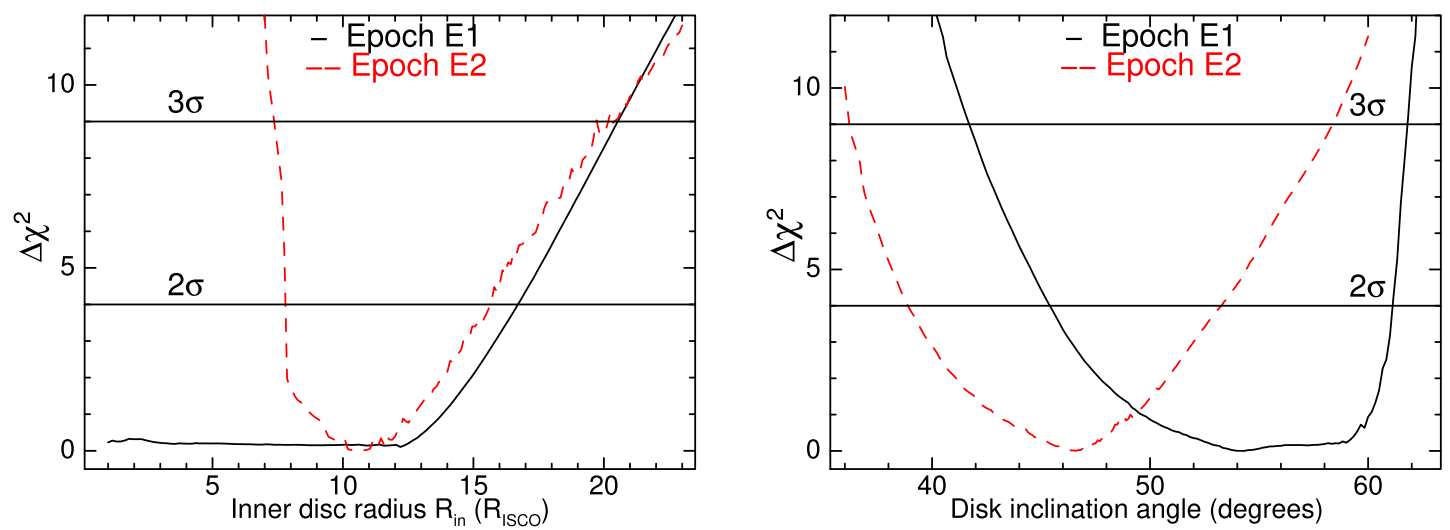

Figure 7. Variations of $\Delta \chi^{2}$ as a function of the inner accretion disc radius ( $R_{\text {in }}$ : in units of $R_{\text {ISCO }}$, determined from the relxilllpCp model) and the disc inclination angle (i). In the left-hand panel, the variation of $\Delta \chi^{2}$ with inner disc radius is shown as observed in Epoch E1 (black solid line) and E2 (red dotted line). It is found that from epoch E1 to E2 within $3 \sigma$ uncertainty, the inner disc radius decreased from $11.92_{-11.92}^{+8.62}\left(R_{\mathrm{ISCO}}\right)$ to $10.39_{-3.02}^{+9.51}\left(R_{\mathrm{ISCO}}\right)$. The right-hand panel shows the variations of $\Delta \chi^{2}$ as a function of disc inclination angle. The inclination angle is found to be $54.19_{-12.48}^{+7.43}{ }^{\circ}$ and $46.59_{-10.38}^{+11.78}{ }^{\circ}$ for $\mathrm{E} 1$ and $\mathrm{E} 2$, respectively, within $3 \sigma$ significance. $\mathrm{a}^{*}$ is fixed at 0.998 .
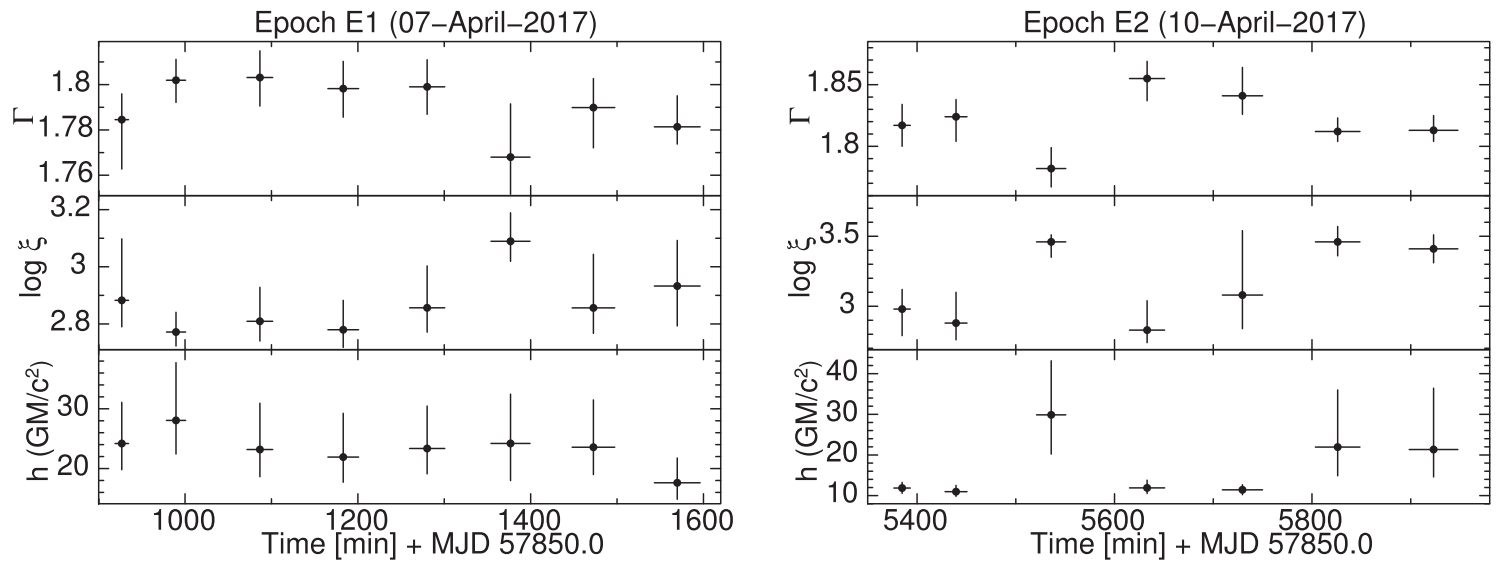

Figure 8. Comparative study of time-resolved energy spectroscopy between E1 and E2. The top panel shows the variation of the photon index ( $\Gamma$ ), the second panel presents the variation of the accretion disc ionization parameter $(\log \xi)$ and the variation of lamp-post height $(h)$ is shown in the third panel. a* is fixed at 0.998 .

where $\bar{x}$ and $\bar{y}$ are the mean of the two series $x$ and $y$.

The $p$-value (significance level) of the correlation is determined by the $T$-test given by

$t=\frac{r}{\sqrt{1-r^{2}}} \sqrt{n-2}$.

As interpreted from the $T$-distribution table, the $p$-values $(p) \leq 0.05$ indicate a strong correlation.

The above-mentioned test reveals a strong correlation between $\Gamma$ and $v_{\mathrm{QPO}}$, as displayed in Fig. 9. The corresponding Pearson's coefficient is also shown in the plot. A strong positive correlation is observed for $\Gamma-v_{\mathrm{QPO}}$ with $\mathrm{r}=0.70\left(p=8.78 \times 10^{-4}\right)$.

\section{DISCUSSION AND CONCLUSIONS}

In this work, we present a broad-band X-ray study of the black hole candidate X-ray binary GRS 1716-249 during its 2016-2017 outburst in the energy range $0.5-79.0 \mathrm{keV}$, using Swift/XRT and NUSTAR FPMA and FPMB data on two different occasions. The joint spectral analysis shows the presence of a broad iron line and reflection hump around $30 \mathrm{keV}$, which can be well modelled with the state-of-art relativistic reflection model relxilllpcp. We

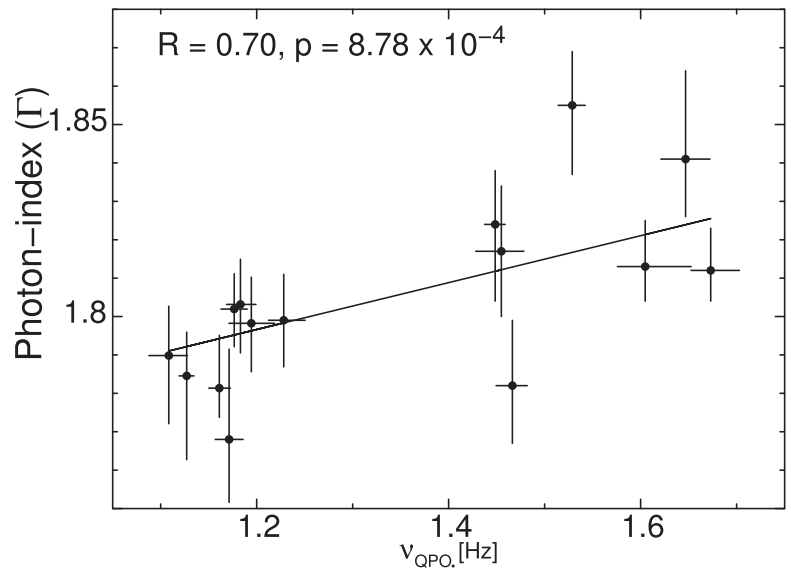

Figure 9. Variation of the photon index $(\Gamma)$ with $\mathrm{QPO}$ frequency. The solid line shows the best-fitting linear dependence. The Pearson $(r)$ correlation coefficients for $v_{\mathrm{QPO}}-\Gamma$ is $0.70\left(p=8.78 \times 10^{-4}\right)$.

constrained the inner disc radius for both epochs and found that the inner disc tends to moves inwards with an increase in the mass 
accretion rate. A low-frequency QPO is observed at $1.20 \pm 0.04 \mathrm{~Hz}$ during the first epoch E1 and the QPO frequency is shifted to $1.55 \pm 0.04 \mathrm{~Hz}$ in the second epoch E2. The time-resolved analysis reveals that there is a hint of variation in the QPO frequency during the second epoch (E2). We observed a strong positive correlation between the QPO frequency and the power-law index.

This work reports for the first time the NuSTAR detection of a LF QPO at $1.20 \pm 0.04 \mathrm{~Hz}$ in the LMXB GRS 1716-249. Earlier studies from the 1993 outbursts of GRS 1716-249 detected a QPOs at $0.04 \mathrm{~Hz}$ which slowly drifted to $0.3 \mathrm{~Hz}$ at the end of the observation (van der Hooft et al. 1996), but there were no QPO detections at frequencies $\geq 1 \mathrm{~Hz}$.

We observed that the QPO frequency ( $v_{\mathrm{QPO}}$ ) increases significantly as the source moves from epoch E1 to E2 and the total flux follows the same trend, which shows a positive correlation between the flux and $v_{\mathrm{QPO}}$ (Ingram \& Done 2011). A positive correlation has also been observed between the photon index and $v_{\mathrm{QPO}}$. The changing QPO frequency could be connected to the inner edge of the accretion disc (Takizawa et al. 1997). If the total flux is connected to the mass accretion rate, this signifies that the inner edge of the accretion disc may move inward with an increasing mass accretion rate.

The correlation between $\Gamma$ and $v_{\mathrm{QPO}}$ has been beautifully demonstrated by the time-resolved spectroscopy over both epochs (E1 and E2). The gradual increase of $\Gamma$ over the total 15 segments (Table 2) from E1 and E2, signifies a gradual successive decrease in the nonthermal emission. The cross-correlation study between $v_{\mathrm{QPO}}$ and the power-law index $\Gamma$ (Fig. 9) clearly establishes a scenario, where the drifting of $v_{\mathrm{QPO}}$ towards higher frequencies and the increase in $\Gamma$, correspond to a decrease of the non-thermal flux (96.97 per cent to 95.45 per cent) with an increase of the disc flux (3.30 per cent to 5.71 per cent) as evident from Table 3 . Therefore, it is evident that the Comptonizing plasma is diminishing with the disc entering into the soft state.

A strong correlation between $\Gamma$ and $v_{\mathrm{QPO}}$ has already been observed in a number of X-ray binaries, for example 4U 1608-52 and 4U $0614+091$ (Kaaret et al. 1998), XTE J1550-564 and GRO J1655-40 (Sobczak et al. 2000), Cyg X-1 (Shaposhnikov \& Titarchuk 2006) and Cyg X-2 (Titarchuk, Kuznetsov \& Shaposhnikov 2007). Sobczak et al. (2000) observed a positive correlation for XTE J1550-564 and a negative correlation for GRO J1655-40. The authors explained that an increase in the mass accretion rate increases the QPO frequency, and the contribution from the power law should be more than 20 per cent for QPOs to be present. Sobczak et al. (2000) have also suggested that the opposite correlation in XTE J1550-564 and GRO J1655-40 could be due to different regions of QPO generation in the two sources. To explain the correlations, Titarchuk \& Fiorito (2004) proposed the transition layer (TL) model. According to this model, a compact bounded coronal region is formed as a natural consequence of the adjustment of the Keplerian disc flow to the innermost sub-Keplerian boundary conditions near the central region. It ultimately ends up forming a TL between the adjustment radius and the innermost boundary. However, this mechanism is unable to produce the inclinationdependent QPOs obtained by Motta et al. (2015).

The observed correlation can be successfully explained on the basis of the Lense-Thirring (LT) precession model (Stella \& Vietri 1998; Ingram et al. 2009) and the truncated disc model (Esin, McClintock \& Narayan 1997; Poutanen, Krolik \& Ryde 1997; Done, Gierliński \& Kubota 2007). When the mass accretion rate shows rapid growth, the truncated disc radius slowly starts moving towards the compact object, hence leading to an increase of the
LT precession frequency. The QPO frequency and its evolution are governed by the size as well as the fluctuations in the truncation radius (increase in QPO frequency represents a decrease of the truncation radius) (Motta et al. 2017). The spectral changes during the source transition can also be explained in this framework. When the mass accretion rate increases, the outer disc gradually starts moving towards the compact object. The disc component becomes stronger and causes greater cooling of the hot inner flow by the cool photons from the disc, resulting in a soft spectrum (Motta et al. 2015; Zhang et al. 2015).

In our spectral analysis, we can estimate the inner radius of the accretion disc $\left(R_{\text {in }}\right)$ in two different ways. The relxilllpcp model directly provides $R_{\text {in }}$, which is found to be $14.78_{-14.78}^{+10.69}\left(r_{\mathrm{g}}\right)$ and $12.88_{-3.74}^{+8.50}\left(r_{\mathrm{g}}\right)$, for epoch E1 and E2, respectively. $R_{\text {in }}$ can be calculated from the normalization of the diskbb model. Using the disc inclination obtained from our spectral analysis, taking the mass to be $4.9 \mathrm{M}_{\odot}$ (Masetti et al. 1996) and the distance to be $2.4 \mathrm{kpc}$ (della Valle et al. 1994), $R_{\text {in }}$ corresponding to epoch $\mathrm{E} 1$ and $\mathrm{E} 2$ found to be $0.66_{-0.13}^{+0.13}\left(r_{\mathrm{g}}\right)$ and $1.12_{-0.13}^{+0.12}\left(r_{\mathrm{g}}\right)$, respectively. We find that the $R_{\text {in }}$ values calculated from the normalization of the diskbb model are smaller than the values estimated from the relxilllpcp model. One possible reason for the discrepancy in the $R_{\text {in }}$ values is that the relxilllpcp model is not self-consistent as it uses the nthComp model with seed photon temperature $\left(T_{\mathrm{bb}}\right)$ fixed at $0.05 \mathrm{keV}$. This value of $T_{\mathrm{bb}}$ is uncharacteristically low for a stellar mass black hole accretion disc. As a result, a higher temperature component becomes necessary to represent a part of the existing cold disc. Secondly, Merloni, Fabian \& Ross (2000) carried out a critical analysis of the usual interpretation of the multicolour disc model parameters for black hole candidates in terms of the inner radius and temperature of the accretion disc. The authors have reported that the diskbb model underestimates the inner disc radius. Merloni et al. (2000) suggested that when the disc contribution is very low and the spectrum is mostly dominated by non-thermal photons, the radius inferred by diskbb is inaccurate. They have also explained that it is very difficult to determine the exact shape of the accretion disc spectrum for the aforementioned case. Kubota et al. (1998) suggested a correction factor to improve the value of $R_{\text {in }}$ calculated from diskbb.

The presence of type $\mathrm{C}$ QPO and the broad-band spectral fitting at both epochs (E1 and E2) confirms that the power-law component dominates over the disc contribution, hence confirming the intermediate state of GRS 1716-249. Here, the soft photons from the accretion disc are Comptonized to higher energies via a hot corona of thermal electrons through the inverse Compton effect (Haardt \& Maraschi 1991, 1993). The two epochs have shown significant spectral changes leading to a successive softening of the source as inferred from the broad-band spectrum at E1 and E2. The broad $\mathrm{Fe} \mathrm{K} \alpha$ line at $6.4 \mathrm{keV}$ is an indication of a spinning black hole (Miller et al. 2009). The observed iron line is extended from nearly $6-8 \mathrm{keV}$ along with a dip around $11 \mathrm{keV}$. This shows that both wings of the Fe $\mathrm{K} \alpha$ line are stretched. It is believed that the broadening of the red wing might be due to Doppler and general relativity (GR) effects, while the blue wing is elongated because of scattering of the photons within the hot inner flow (Fabian et al. 2000; Miller 2007; Miller et al. 2017).

Reflection features have already been detected in a number of X-ray binaries. Done \& Życki (1999) have significantly detected the relativistic smearing of the reflected spectrum in Cyg X-1. Using the X-ray spectrum, they have simultaneously constrained the ionization state and the percentage of relativistic smearing. They analysed observations from three different missions namely: 
EXOSAT GSPC, Ginga, and ASCA. They implemented the PEXRIV model in XSPEC to fit the reflection spectrum, and the DISCLINE model in XSPEC is used to account for the relativistic smearing. They fitted the spectrum for different values of the inclination angle and iron abundance. During the entire analysis, the photon index ranged from 1.44 to 1.91 . They also reported $\xi$ and $R_{\text {in }}$ for various observations. Our results are found to be consistent with their results, and during our observations, $\Gamma$ varies from 1.80 to 1.93. On many occasions, the values of the inner disc radius and accretion disc ionization parameter as reported by Done \& Życki (1999), are found to be close to the values observed in this work.

In a separate study, Miller et al. (2002) resolved the Fe K $\alpha$ line region in Cyg X-1 through spectral analysis. The Chandra $\mathrm{X}$-ray observatory detected the source in an intermediate spectral state during these observations. The authors observed a narrow line around $6.42 \mathrm{keV}$ along with a broad-line feature at $5.82 \mathrm{keV}$ and a smeared edge at $7.3 \mathrm{keV}$. These results support our findings, as the study reported a photon index around 1.8 which is close to the value we found during E1. The value of $R_{\text {in }}$ reported in that study is similar to the value of $R_{\text {in }}$ found during E1 in this work. Two different lines and a smeared edge are not observed in our study, either because of the superior spectral resolution of Chandra in comparison to NuSTAR (Canizares et al. 2000; Harrison et al. 2013), or because they may not be present in GRS 1716-249.

Recently, there have been several attempts to find a correlation between QPOs and iron lines to constrain the origin of the QPOs (Miller \& Homan 2005; Ingram \& van der Klis 2015). The basic idea behind such an attempt is that both the QPOs and Fe line possibly originate from the inner part of the accretion disc. Therefore, they can provide an independent signature of the geometry of the inner disc. It has been found that the variation in the QPO frequency and the QPO phase is correlated with the variation of the iron line centroid frequency (Ingram et al. 2016, 2017). These studies reflect the geometric origin of QPOs. However, our current findings are limited to explore any such correlations between QPO and Fe line because of the limited extent of our observations. In future work, we plan to use better timing and multi-epoch data from ASTROSAT (Antia et al. 2017; Verdhan Chauhan et al. 2017), spanning over several months, along with our current results, to study the QPOs with more physical explanations and rigorously updated physical models.

\section{ACKNOWLEDGEMENTS}

This research has made use of the software and/or data obtained through the High Energy Astrophysics Science Archive Research Center (HEASARC) online service, provided by the NASA/Goddard Space Flight Center and the SWIFT data center. We thank NUSTAR team for making NUSTAR data public. MAXI data are obtained from MAXI team, RIKEN, JAXA. The authors gratefully acknowledge the anonymous referee for constructive comments that improved the paper. The authors are also thankful to PI's for proposing these observations. JC and SC are also grateful to Prof. H. M. Antia, Prof. A. R. Rao, and Prof. S. Bhattacharyya for constructive discussions about diagnostics and interpretations. The work by MB is supported by the Department of Science and Technology and National Research Foundation ${ }^{4}$ (NRF) of

\footnotetext{
${ }^{4}$ Any opinion, finding and conclusion or recommendation expressed in this material is that of the author and the NRF does not accept any liability in this regard.
}

South Africa through the South African Research Chairs Initiative (SARChI), grant no. 64789. SC acknowledges the supports by CSRNWU and NRF.

\section{REFERENCES}

Antia H. M. et al., 2017, ApJS, 231, 10

Armas Padilla M., Muñoz-Darias T., 2017, Astron. Telegram, 10236

Arnaud K. A., 1996, in Jacoby G. H., Barnes J., eds, ASP Conf. Ser. Vol. 101, Astronomical Data Analysis Software and Systems V. Astron. Soc. Pac., San Francisco, p. 17

Asplund M., Grevesse N., Sauval A. J., Scott P., 2009, ARA\&A, 47, 481

Bachetti M. et al., 2015, ApJ, 800, 109

Ballet J., Denis M., Gilfanov M., Sunyaev R., Harmon B. A., Zhang S. N., Paciesas W. S., Fishman G. J., 1993, IAU Circ., 5874

Bardeen J. M., Press W. H., Teukolsky S. A., 1972, ApJ, 178, 347

Bassi T. et al., 2019, MNRAS, 482, 1587

Bassi T., Del Santo M., Motta S. E., 2017, Astron. Telegram, 10371

Belloni T., Klein-Wolt M., Méndez M., van der Klis M., van Paradijs J., 2000, A\&A, 355, 271

Belloni T. M., Stella L., 2014, SSRv, 183, 43

Canizares C. R. et al., 2000, ApJ, 539, L41

Casella P., Belloni T., Stella L., 2005, ApJ, 629, 403

Choudhury K., García J. A., Steiner J. F., Bambi C., 2017, ApJ, 851, 57

Dauser T., García J., Parker M. L., Fabian A. C., Wilms J., 2014, MNRAS, 444, L100

della Valle M., Mirabel I. F., Rodriguez L. F., 1994, A\&A, 290, 803

Done C., Życki P. T., 1999, MNRAS, 305, 457

Done C., Gierlińnski M., Kubota A., 2007, A\&AR, 15, 1

Esin A. A., McClintock J. E., Narayan R., 1997, ApJ, 486, 865

Fabian A. C., Iwasawa K., Reynolds C. S., Young A. J., 2000, PASP, 112, 1145

García J., Dauser T., Reynolds C. S., Kallman T. R., McClintock J. E., Wilms J., Eikmann W., 2013, ApJ, 768, 146

García J. et al. 2014, ApJ, 782, 76

Haardt F., Maraschi L., 1991, ApJ, 380, L51

Haardt F., Maraschi L., 1993, ApJ, 413, 507

Harmon B. A., Fishman G. J., Paciesas W. S., Zhang S. N., 1993, IAU Circ., 5900

Harrison F. A. et al., 2013, ApJ, 770, 103

Homan J., Wijnands R., van der Klis M., Belloni T., van Paradijs J., KleinWolt M., Fender R., Méndez M., 2001, ApJS, 132, 377

Ingram A., Done C., 2011, MNRAS, 415, 2323

Ingram A., van der Klis M., 2015, MNRAS, 446, 3516

Ingram A., Done C., Fragile P. C., 2009, MNRAS, 397, L101

Ingram A., van der Klis M., Middleton M., Done C., Altamirano D., Heil L., Uttley P., Axelsson M., 2016, MNRAS, 461, 1967

Ingram A., van der Klis M., Middleton M., Altamirano D., Uttley P., 2017, MNRAS, 464, 2979

Kaaret P., Yu W., Ford E. C., Zhang S. N., 1998, ApJ, 497, L93

Kalberla P. M. W., Burton W. B., Hartmann D., Arnal E. M., Bajaja E., Morras R., Pppel W. G. L., 2005, A\&A, 440, 775

Kubota A., Tanaka Y., Makishima K., Ueda Y., Dotani T., Inoue H., Yamaoka K., 1998, PASJ, 50, 667

Ling J. C., Wheaton W. A., 2005, ApJ, 622, 492

Madsen K. K. et al., 2015, ApJS, 220, 8

Makishima K. et al., 1986, ApJ, 308, 635

Marcotulli L. et al., 2017, ApJ, 839, 96

Masetti N., Bianchini A., Bonibaker J., della Valle M., Vio R., 1996, A\&A, 314,123

Masumitsu T. et al., 2016, Astron. Telegram, 9895

Merloni A., Fabian A. C., Ross R. R., 2000, MNRAS, 313, 193

Miller J. M., Reynolds C. S., Fabian A. C., Miniutti G., Gallo L. C., 2009, ApJ, 697, 900

Miller J. M., Parker M. L., Fuerst F., Bachetti M., Barret D., Grefenstette B. W. et al., 2013, ApJ, 779, L2

Miller J. M. et al., 2002, ApJ, 578, 348 
Miller J. M. et al., 2017, Astron. Telegram, 10296

Miller J. M., 2007, ARA\&A, 45, 441

Miller J. M., Homan J., 2005, ApJ, 618, L107

Mitsuda K. et al., 1984, PASJ, 36, 741

Morihana K. et al., 2013, PASJ, 65, L10

Motta S., Muñoz-Darias T., Casella P. et al., 2011, MNRAS, 418, 2292

Motta S. E., Muñoz-Darias T., Sanna A., Fender R., Belloni T., Stella L., 2014b, MNRAS, 439, L65

Motta S. E., Casella P., Henze M., Muñoz-Darias T., Sanna A., Fender R., Belloni T., 2015, MNRAS, 447, 2059

Motta S. E., Rouco-Escorial A., Kuulkers E., Muñoz-Darias T., Sanna A., 2017, MNRAS, 468, 2311

Negoro H. et al., 2016, Astron. Telegram, 9876

Pahari M., Yadav J. S., Bhattacharyya S., 2014, ApJ, 783, 141

Pahari M., Misra R., Dewangan G. C., Pawar P., 2015, ApJ, 814, 158

Parker M. L. et al., 2015, ApJ, 808, 9

Pearson K., 1920, Biometrika, 13, 25

Poutanen J., Krolik J. H., Ryde F., 1997, MNRAS, 292, L21

Remillard R. A., McClintock J. E., 2006, ARA\&A, 44, 49

Remillard R. A., Muno M. P., McClintock J. E., Orosz J. A., 2002, ApJ, 580, 1030

Revnivtsev M. et al., 1996, MPE Rep., 263, 185

Romano P. et al., 2006, A\&A, 456, 917

Ross R. R., Fabian A. C., 1993, MNRAS, 261, 74
Ross R. R., Fabian A. C., 2005, MNRAS, 358, 211

Shaposhnikov N., Titarchuk L., 2006, ApJ, 643, 1098

Sobczak G. J., McClintock J. E., Remillard R. A., Cui W., Levine A. M., Morgan E. H., Orosz J. A., Bailyn C. D., 2000, ApJ, 531, 537

Stella L., Vietri M., 1998, ApJ, 492, L59

Takizawa M. et al., 1997, ApJ, 489, 272

Thorne K. S., 1974, ApJ, 191, 507

Titarchuk L., Fiorito R., 2004, ApJ, 612, 988

Titarchuk L., Kuznetsov S., Shaposhnikov N., 2007, ApJ, 667, 404

van der Hooft F. et al., 1999, ApJ, 519, 332

van der Hooft F. et al., 1996, A\&AS, 120, 141

van der Klis M., 1989, ARA\&A, 27, 517

Vaughan S., 2005, A\&A, 431, 391

Verdhan Chauhan J. et al., 2017, ApJ, 841, 41

Wijnands R., van der Klis M., 1999, ApJ, 514, 939

Wilms J., Allen A., McCray R., 2000, ApJ, 542, 914

Zdziarski A. A., Johnson W. N., Magdziarz P., 1996, MNRAS, 283, 193

Zhang L., Chen L., Qu J.-1., Bu Q.-c., 2015, AJ, 149, 82

Zẏcki P. T., Done C., Smith D. A., 1999, MNRAS, 309, 561

This paper has been typeset from a $\mathrm{T}_{\mathrm{E}} \mathrm{X} / \mathrm{LT} \mathrm{E} \mathrm{X}$ file prepared by the author. 